\title{
Classification of Hyperbolic Singularities in Interval 3-Dimensional Linear Differential Systems
}

\author{
Marina Tuyako Mizukoshi ${ }^{1(\bowtie)}\left(\mathbb{D}\right.$, Alain $\operatorname{Jacquemard}^{2(\bowtie)}$, \\ and Weldon Alexander Lodwick ${ }^{3(\bowtie)}$ (D) \\ 1 Instituto de Matemática e Estatística, Universidade Federal de Goiás, \\ Campus II - Samambaia, Goiânia, GO 74690-900, Brazil \\ tuyako@ufg.br \\ 2 Université Bourgogne Franche-Comté, Institut de Mathématiques de Bourgogne, \\ UMR 5584, 21000 Dijon, France \\ Alain. Jacquemard@u-bourgogne.fr \\ 3 Department of Mathematical and Statistical Sciences, University of Colorado, \\ 1201 Larimer Street, Denver, CO 80204, USA \\ Weldon.Lodwick@ucdenver.edu \\ http://www.ime.ufg.br, https://math.u-bourgogne.fr/, \\ https://clas.ucdenver.edu/mathematical-and-statistical-sciences
}

\begin{abstract}
We study the classification of the hyperbolic singularities to 3-dimensional interval linear differential equations as an application of interval eigenvalues using the Constraint Interval Arithmetic (CIA). We also present the ideas to calculate the interval eigenvalues using the standard interval arithmetic.
\end{abstract}

Keywords: Interval eigenvalues $\cdot$ Dynamical systems $\cdot$ Classification of singularities

\section{Introduction}

Many applied problems have uncertainties or inaccuracies due to data measurement errors, lack of complete information, simplification assumption of physical models, variations of the system, and computational errors. An encoding of uncertainty as intervals instead of numbers when applicable is an efficient way to address the aforementioned challenges.

When studying an interval problem we need to first understand what is the context. In this presentation, we are concerned if there exists dependence, independence or both in the parameters involved. In accordance to this context we need to choose the appropriated arithmetic. Where we have the total independence or dependence, we can use interval arithmetic or single level arithmetic 
(SLA). If we are studying a problem where there is the independence as well as the dependence in parameters then constraint interval arithmetic (CIA) is a good choice.

We are interested in studying the interval eigenvalue problem associated with differential equations. This problem has many applications in the fields of mechanics and engineering. The first interval eigenvalues results were obtained by Deif [5], Deif and Rohn [15], Rohn [16]. Subsequently, approximation methods results were obtained by Qiu et al. [14], Leng et al. [10], Hladik [9] and Hladik et al. [6-8].

This presentation establishes conditions on the parameters of interval linear autonomous differential systems to classify the hyperbolic equilibrium point in 3-dimensions. Moreover a detailed study is given for an example using CIA along with a computational method for complex conjugate eigenvalues where we obtain the lower and upper bounds of the real eigenvalue.

\section{Preliminaries}

The following outlines the standard interval arithmetic WSMA (Warmus, Sunaga and Moore Arithmetic) and CIA (constraint interval arithmetic).

Let $\mathbf{x}=\left[\begin{array}{ll}\underline{x} & \bar{x}\end{array}\right] \quad \mathbf{y}=\left[\begin{array}{ll}y & \bar{y}\end{array}\right]$, be such that $\underline{x} \leq \bar{x}$ and $\underline{y} \leq \bar{y}$, then for WSMA arithmetic we have the following operations:

1. $\mathbf{x}+\mathbf{y}=[\underline{x}+\underline{y} \bar{x}+\bar{y}]$

2. $\mathbf{x}-\mathbf{y}=[\underline{x}-\overline{\bar{y}} \bar{x}-\underline{y}]$;

3. $\mathbf{x} \times \mathbf{y}=[\min \{\underline{x} \underline{y}, \underline{x} \bar{y}, \overline{x y}, \underline{x} \bar{y}\} \max \{\underline{x} \underline{y}, \underline{x} \bar{y}, \overline{x y}, \underline{x} \bar{y}\}]$

4. $\mathbf{x} \div \mathbf{y}=[\min \{\underline{x} \div \underline{y}, \bar{x} \div \bar{y}, \underline{x} \div \bar{y}, \bar{x} \div \underline{y}\} \max \{\underline{x} \div \underline{y}, \bar{x} \div \bar{y}, \underline{x} \div \bar{y}, \bar{x} \div \underline{y}\}], 0 \notin[\underline{y} \bar{y}]$.

Remark: Note that in WSMA arithmetic $\mathbf{x}-\mathbf{x}$ is never 0 unless $\mathbf{x}$ is a real number (width zero) nor is $\mathbf{x} \div \mathbf{x}=1$.

Definition 1. [11] An interval $[\underline{x} \bar{x}] C I$ (constraint interval) representation is the real single-valued function $\boldsymbol{x}(\gamma)=\gamma \bar{x}+(1-\gamma) \underline{x}, 0 \leq \gamma \leq 1$. Constraint interval arithmetic (CIA) is $\boldsymbol{z}=\boldsymbol{x} \circ \boldsymbol{y}$, where $\boldsymbol{z}=[\underline{z} \bar{z}]=\left\{z\left(\gamma_{1}, \gamma_{2}\right) ; z\left(\gamma_{1}, \gamma_{2}\right)=\right.$ $\left.\left(\gamma_{1} \bar{x}+\left(1-\gamma_{1}\right) \underline{x}\right) \circ\left(\gamma_{2} \bar{y}+\left(1-\gamma_{2}\right) \underline{y}\right), 0 \leq \gamma_{1} \leq 1,0 \leq \gamma_{2} \leq 1\right\}$, and $\underline{z}=$ $\min \left\{z\left(\gamma_{1}, \gamma_{2}\right)\right\}, \bar{z}=\max \left\{z\left(\gamma_{1}, \gamma_{2}\right)\right\}, \circ \in\{+,-, \times, \div\}$.

The set of $m \times n$ interval matrices will be denoted by $\mathbb{I R}^{m \times n}$. An interval matrix $\mathbf{A}=\left(\mathbf{A}_{i k}\right)$ is interpreted as a set of real $m \times n$ matrices

$$
\mathbf{A}=\left\{A \in \mathbb{R}^{m \times n} ; A_{i k} \in\left(\mathbf{A}_{i k}\right) \text { for } i=1, \ldots, m, k=1, \ldots, n\right\} .
$$

Denote by $\mathbf{A}=\left[\begin{array}{ll}\underline{A} & \bar{A}\end{array}\right]$, where $\underline{A}$ and $\bar{A}$ are matrix whose entries are given by right and left sides of all intervals numbers $\left(\mathbf{a}_{i k}\right) \in \mathbf{A}$, respectively. In the CI context each element in $\mathbf{A}$ is given by $\mathbf{a}_{i j}\left(\gamma_{i j}\right)=\underline{a}_{i j}+\gamma_{i j} w_{\mathbf{a}_{i j}}, 1 \leq i \leq n, 1 \leq$ $j \leq m, w_{\mathbf{a}_{i j}}=\bar{a}_{i j}-\underline{a}_{i j}, \gamma_{i} \in[0,1]$. 
Definition 2. [5] Given $[A]=[\underline{A} \bar{A}]$, an interval matrix in $\mathbb{I R}^{n \times n}$, the set of eigenvalues is given by:

$$
\Lambda([A])=\{\lambda ; A x=\lambda x, x \neq 0, A \in[A]\} .
$$

In addition, we denote by $A_{c}=\frac{1}{2}(\underline{A}+\bar{A}), \triangle_{A}=\frac{1}{2}(\underline{A}-\bar{A})$, the midpoint and the radius of $[A]$, respectively.

In what follows we use the notation $\vec{\gamma}$ to mean the dependence of the choice of the values for $\gamma_{i j}, i, j=1, \ldots, n$ in the interval $\left[\begin{array}{ll}0 & 1\end{array}\right]$.

Definition 3. [12] Let be an interval matrix $\boldsymbol{A}=[\underline{A} \quad \bar{A}]$, then the CI matrix is defined by

$$
A(\vec{\gamma})=\left(\begin{array}{ccc}
\underline{a}_{11}+\gamma_{11} w_{a_{11}} & \ldots & \underline{a}_{1 n}+\gamma_{1 n} w_{a_{1 n}} \\
\ldots & \ldots & \ldots \\
\underline{a}_{n 1}+\gamma_{n 1} w_{a_{n 1}} & \ldots & \underline{a}_{n n}+\gamma_{n n} w_{a_{n n}}
\end{array}\right)=\underline{A}+\Gamma \odot W,
$$

where $\underline{A}=\left(\underline{a}_{i j}\right), W=\left(w_{a_{i j}}\right)=\left(\bar{a}_{i j}-\underline{a}_{i j}\right), \Gamma=\left(\gamma_{i j}\right), 0 \leq \gamma_{i j} \leq 1$, for $i=1, \ldots, n$ and $j=1, \ldots, n$ and the symbol $\odot$ denotes componentwise multiplication. Then, we say that $\lambda(\Gamma)$ is an eigenvalue of $A(\vec{\gamma})$ if $\exists x \neq 0 \mid A(\vec{\gamma}) x=\lambda(\vec{\gamma})$ i.e., $\left.\operatorname{det}(A(\vec{\gamma}))-\lambda(\vec{\gamma}) I_{n}\right)=0$, where $I_{n}$ is the identity matrix of order $n$.

Remark: Here for each choice of matrix $\Gamma$ we have a deterministic problem to calculate eigenvalues. We can get the interval eigenvalues by minimizing and maximizing $\lambda(\vec{\gamma})$ by varying all $\gamma_{i j}, i, j=1, \ldots, n$ between 0 and 1 .

To classify the equilibrium point in a 3 -dimensional linear differential system, firstly we need to know how we can classify it in according the eigenvalues obtained of the matrix of the coefficients from linear differential system.

Consider a linear three-dimensional autonomous systems $X^{\prime}(t)=$ $A X(t), X(t), X^{\prime}(t) \in M_{3 \times 1}(\mathbb{R}), A \in M_{3 \times 3}(\mathbb{R})$ of the form

$$
\left\{\begin{array}{l}
x^{\prime}(t)=a_{11} x+a_{12} y+a_{13} z \\
y^{\prime}(t)=a_{21} x+a_{22} y+a_{23} z \\
z^{\prime}(t)=a_{31} x+a_{32} y+a_{33} z
\end{array}\right.
$$

where the $a_{i j}$ are constants. Suppose that (1) satisfies the existence and uniqueness theorem. Given a matrix of order $3 \times 3$, we have the following possibilities for the real canonical forms:

$$
\left(\begin{array}{ccc}
\lambda_{1} & 0 & 0 \\
0 & \lambda_{2} & 0 \\
0 & 0 & \lambda_{3}
\end{array}\right), \quad\left(\begin{array}{ccc}
\lambda_{1} & 1 & 0 \\
0 & \lambda_{1} & 0 \\
0 & 0 & \lambda_{3}
\end{array}\right), \quad\left(\begin{array}{ccc}
\lambda_{1} & 1 & 0 \\
0 & \lambda_{1} & 1 \\
0 & 0 & \lambda_{1}
\end{array}\right), \quad\left(\begin{array}{ccc}
\alpha-\beta & 0 \\
\beta & \alpha & 0 \\
0 & 0 & \lambda_{3}
\end{array}\right),
$$

where the eigenvalue $\lambda=\alpha \pm i \beta$ with $\alpha=0$ for pure imaginary, $\beta=0$ for real case and both are different of zero for complex $\lambda$. If the singularities in matrix $A$ are hyperbolic $\alpha \neq 0$ then we have the following possibilities: 
Table 1. Classification of the hyperbolic flows in dimension 3.

\begin{tabular}{l|l}
\hline Classification & Eigenvalues $\lambda_{1}, \lambda_{2}, \lambda_{3}$ \\
\hline Attractors (stable) & $\lambda=\alpha \pm i \beta(\alpha<0)$ and $\lambda_{3}<0$ or $\lambda_{1}, \lambda_{2}, \lambda_{3}<0$ \\
\hline Saddle point & $\begin{array}{l}\lambda=\alpha \pm i \beta, \alpha<0(>0) \text { and } \lambda_{3}>0(<0) \text { or } \\
\lambda_{1}, \lambda_{2}<0(>0) \text { and } \lambda_{3}>0(<0)\end{array}$ \\
\hline Repellors (unstable) & $\lambda=\alpha \pm i \beta(\alpha>0)$ and $\lambda_{3}>0$ or $\lambda_{1}, \lambda_{2}, \lambda_{3}>0$ \\
\hline
\end{tabular}

Remark: We are not interested in the cases $\alpha=0$ and/or the real eigenvalue equal to zero since we cannot classify the equilibrium point.

\section{Interval 3-Dimensional Linear Differential System}

Given system (1) with the initial conditions, we can to consider the Initial Value Problem with uncertainty, where the initial conditions and/or coefficients are uncertainty. The behavior of the solution trajectories are not changed if only its initial condition has a small perturbation. For example, consider the system
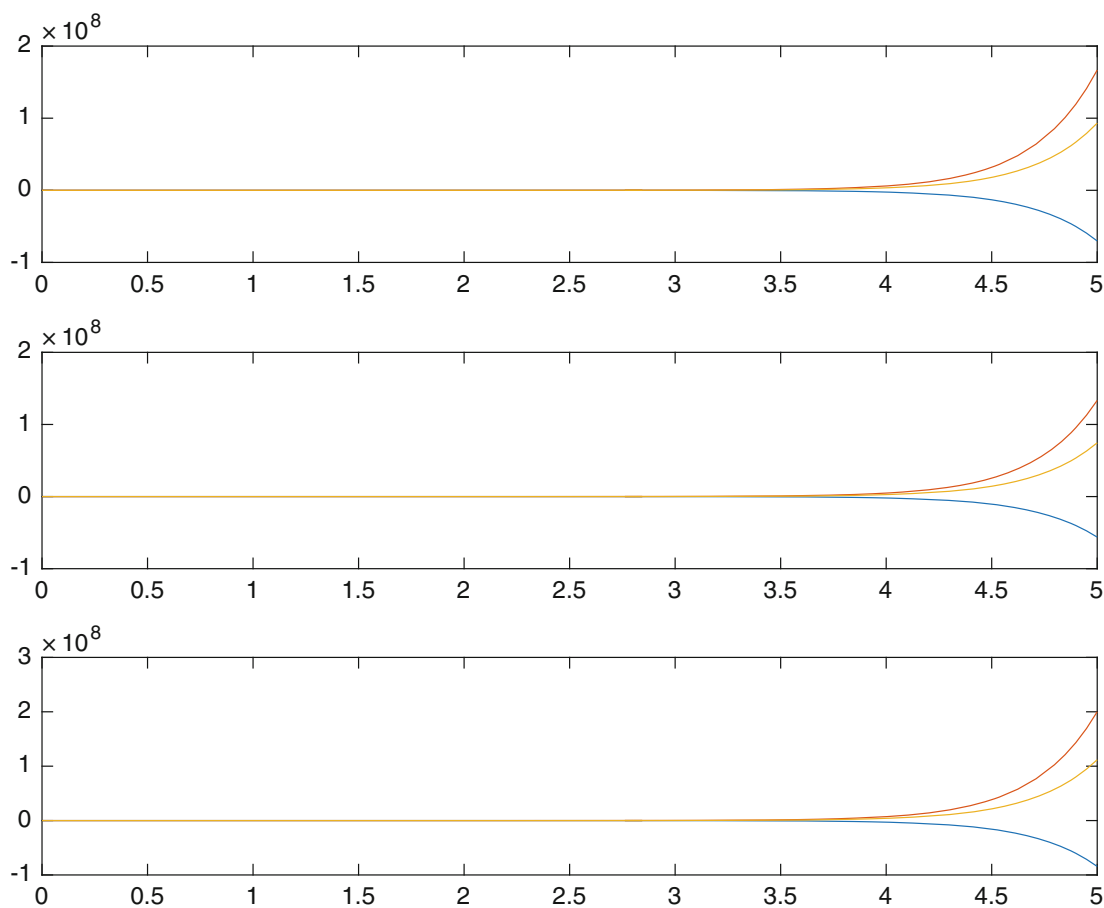

Fig. 1. The graph for $x(t), y(t), z(t)$ com initial conditions [0.8 0.8 0.8], [ $\left.\begin{array}{lll}1 & 1 & 1\end{array}\right]$ and [ $\left.\begin{array}{lll}1.2 & 1.2 & 1.2\end{array}\right]$, respectively. 
$X^{\prime}(t)=A X(t)$, where $A=\left(\begin{array}{ccc}2 & 0 & -1 \\ 3 & 4 & 1 \\ 2 & 1 & 3\end{array}\right)$ with the initial conditions $\mathbf{x}_{0}=\mathbf{y}_{0}=$ $\mathbf{z}_{0}=\left[\begin{array}{ll}0.8 & 1.2\end{array}\right]$ then we have the following unstable trajectories (see Fig. 1).

Remark: When only the initial conditions are intervals then the eigenvalues do not change and the stability or instability is kept. But, if in (1) the entries in the matrix of coefficient vary, then we need to evaluate what will happen with the equilibrium point in (1).

Then, we consider in (1), $A$ as an interval matrix. According to Definition 3, we have the following problem:

$$
\left\{\begin{array}{l}
x^{\prime}(t)=\left(\underline{a}_{11}+\gamma_{11} w_{11}\right) x(t)+\left(\underline{a}_{12}+\gamma_{12} w_{12}\right) y(t)+\left(\underline{a}_{13}+\gamma_{13} w_{13}\right) z(t) \\
y^{\prime}(t)=\left(\underline{a}_{21}+\gamma_{21} w_{21}\right) x(t)+\left(\underline{a}_{22}+\gamma_{22} w_{22}\right) y(t)+\left(\underline{a}_{23}+\gamma_{23} w_{23}\right) z(t) \\
z^{\prime}(t)=\left(\underline{a}_{31}+\gamma_{31} w_{31}\right) x(t)+\left(\underline{a}_{32}+\gamma_{32} w_{32}\right) y(t)+\left(\underline{a}_{33}+\gamma_{33} w_{33}\right) z(t)
\end{array}\right.
$$

where $\gamma_{i j} \in[0,1], w_{i j}=\bar{a}_{i j}-\underline{a}_{i j}$, for $i, j=1,2,3$.

Observe that system (2):

1. Has an unique equilibrium point at the origin $(0,0,0)$ if given the matrix $A(\vec{\gamma})=\left(\begin{array}{lll}\underline{a}_{11}+\gamma_{11} w_{11} & \underline{a}_{12}+\gamma_{12} w_{12} & \underline{a}_{13}+\gamma_{13} w_{13} \\ \underline{a}_{21}+\gamma_{21} w_{21} & \underline{a}_{22}+\gamma_{22} w_{22} & \underline{a}_{22}+\gamma_{23} w_{23} \\ \underline{a}_{31}+\gamma_{31} w_{31} & \underline{a}_{32}+\gamma_{32} w_{32} & \underline{a}_{33}+\gamma_{33} w_{33}\end{array}\right)$, the $\operatorname{det}(A(\vec{\gamma})) \neq 0, \forall \gamma_{i j} \in[0,1]$ for $i, j=1,2,3$.

2. For $\vec{\gamma}=\left(\gamma_{11}, \gamma_{12}, \gamma_{13}, \gamma_{21}, \gamma_{22}, \gamma_{23}, \gamma_{31}, \gamma_{32}, \gamma_{33}\right)$, the eigenvalues are obtained from the equation:

$$
p(\lambda(\vec{\gamma}))=-\lambda^{3}(\vec{\gamma})+a_{2}(\vec{\gamma}) \lambda^{2}(\vec{\gamma})-a_{1}(\vec{\gamma}) \lambda(\vec{\gamma})+a_{0}(\vec{\gamma}),
$$

where

$$
\begin{aligned}
& a_{2}(\vec{\gamma})=\operatorname{tr}(A(\Gamma))=\underline{a}_{11}+\gamma_{11} w_{11}+\underline{a}_{22}+\gamma_{22} w_{22}+\underline{a}_{33}+\gamma_{33} w_{33} ; \\
& a_{1}(\vec{\gamma})=\left[-\left(\underline{a}_{11}+\gamma_{11} w_{11}\right)-\left(\underline{a}_{22}+\gamma_{22} w_{22}\right)-\right]\left(\underline{a}_{33}+\gamma_{33} w_{33}\right)+\left[\underline{a}_{31}+\gamma_{31} w_{31}+\right. \\
& \left.\left.\underline{a}_{13}+\gamma_{13} w_{13}\right]\right]\left(\underline{a}_{32}+\gamma_{32} w_{32}\right)+\left(\underline{a}_{12}+\gamma_{12} w_{12}\right)\left(\underline{a}_{13}+\gamma_{13} w_{13}\right)-\left(\underline{a}_{11}+\gamma_{11} w_{11}\right)\left(\underline{a}_{22}+\right. \\
& \left.\gamma_{22} w_{22}\right) ; \\
& a_{0}(\vec{\gamma})=\left[\left(\underline{a}_{11}+\gamma_{11} w_{11}\right)\left(\underline{a}_{22}+\gamma_{22} w_{22}\right)-\left(\underline{a}_{12}+\gamma_{12} w_{12}\right)\left(\underline{a}_{13}+\gamma_{13} w_{13}\right)\left(\underline{a}_{21}+\right.\right. \\
& \left.\left.\gamma_{21} w_{21}\right)\right]\left(\underline{a}_{33}+\gamma_{33} w_{33}\right)+\left[\left[\underline{a}_{12}+\gamma_{12} w_{12}-\underline{a}_{11}-\gamma_{11} w_{11}\right]\left(\underline{a}_{31}+\gamma_{31} w_{31}\right)+\left[\underline{a}_{21}+\right.\right. \\
& \left.\left.\gamma_{21} w_{21}-\underline{a}_{22}-\gamma_{22} w_{22}\right]\left(\underline{a}_{13}+\gamma_{13} w_{13}\right)\right]\left(\underline{a}_{32}+\gamma_{32} w_{32}\right) .
\end{aligned}
$$

Theorem 1. If system (2) has a unique equilibrium point at $(0,0,0)$, then there is a matrix $\Gamma=\left(\gamma_{i j}\right), i, j=1,2,3$ such that the equilibrium point is classified according to Table 1.

Proof. Given the matrix $A(\vec{\gamma})$ in system (2), the nature of the equilibrium is defined according to the zeroes of the characteristic polynomial of $A(\vec{\gamma})$

$$
-\lambda^{3}(\vec{\gamma})+a_{2}(\vec{\gamma}) \lambda^{2}(\vec{\gamma})+a_{1}(\vec{\gamma}) \lambda(\vec{\gamma})+a_{3}(\vec{\gamma})=0,
$$


The roots of the polynomial of order 3 in (4) are defined according to the following discriminant [4]:

$$
\triangle(\vec{\gamma})=4 p^{3}(\vec{\gamma})+q^{2}(\vec{\gamma})
$$

where $q(\vec{\gamma})=a_{0}(\vec{\gamma})+\frac{a_{2}(\vec{\gamma}) a_{1}(\vec{\gamma})}{3}+\frac{2}{27} a_{2}^{3}(\vec{\gamma})$ and $p(\vec{\gamma})=-\frac{1}{3} a_{1}(\vec{\gamma})-$ $\frac{1}{9} a_{2}^{2}(\vec{\gamma})$. Then, we have:

1. If $\triangle(\vec{\gamma})<0$, (4) has 3 different real roots;

2. If $\triangle(\vec{\gamma})>0$, (4) has 1 real root and 2 complex (conjugate) roots;

3. If $\triangle(\vec{\gamma})=0$ and $p(\vec{\gamma})<0$ then (4) has 3 real roots, where two of them are equal;

4. If $p(\vec{\gamma})=q(\vec{\gamma})=0$, then (4) has 3 equal real roots.

The analysis depends of the entries in matrix $A(\vec{\gamma})$ for $\gamma_{i j}, i, j=1,2,3$.

1. First case: if all entries of matrix $A(\vec{\gamma})$ in system (2) are dependent, then $\gamma_{i j}=\gamma, \forall i, j=1,2,3$ and the eigenvalues are obtained from the equation:

$p(\lambda(\gamma))=-\lambda^{3}(\gamma)+a_{2}(\gamma) \lambda^{2}(\gamma)-a_{1}(\gamma) \lambda(\gamma)+a_{0}(\gamma)$,

where

$a_{2}(\gamma)=\operatorname{tr}(A(\gamma))=\underline{a}_{11}+\underline{a}_{22}+\underline{a}_{33}+\gamma\left(w_{11}+w_{22}+w_{33}\right)$;

$a_{1}(\gamma)=\left|\begin{array}{ll}\underline{a}_{11}+\gamma w_{11} & \underline{a}_{12}+\gamma w_{12} \\ \underline{a}_{21}+\gamma w_{21} & \underline{a}_{22}+\gamma w_{22}\end{array}\right|+\left|\begin{array}{ll}\underline{a}_{11}+\gamma_{1} w_{11} & \underline{a}_{13}+\gamma_{3} w_{13} \\ \underline{a}_{31}+\gamma_{7} w_{31} & \underline{a}_{33}+\gamma_{9} w_{33}\end{array}\right|+\left|\begin{array}{ll}\underline{a}_{22}+\gamma_{5} w_{22} & \underline{a}_{22}+\gamma_{6} w_{23} \\ \underline{a}_{32}+\gamma_{8} w_{32} & \underline{a}_{33}+\gamma_{9} w_{33}\end{array}\right| ;$

$a_{0}(\gamma)=\operatorname{det}(A(\gamma))$.

Then, the classification can be obtained using the particular expression (5).

2. Second case: if the matrix $A(\vec{\gamma})$ is symmetric, then $\underline{a}_{i j}+\gamma i j w_{i j}=\underline{a}_{j i}+$ $\gamma_{j i} w_{j i}, \forall i \neq j, i, j=1,2,3$ and $\vec{\gamma}=\left(\gamma_{11}, \gamma_{12}, \gamma_{13}, \gamma_{22}, \gamma_{23}, \gamma_{33}\right)$. In this case, the eigenvalues are obtained from the equation:

$p\left(\lambda(\vec{\gamma})=-\lambda^{3}(\vec{\gamma})+\lambda^{2}(\vec{\gamma}) a_{2}(\vec{\gamma})-\lambda(\vec{\gamma}) a_{1}(\vec{\gamma})+a_{0}(\vec{\gamma})\right.$,

where

$\left.a_{2}(\vec{\gamma})=\operatorname{tr}(A(\vec{\gamma}))=\underline{a}_{11}+\underline{a}_{22}+\underline{a}_{33}+\gamma_{11} w_{11}+\gamma_{22} w_{22}+\gamma_{33} w_{33}\right) ;$

$a_{1}(\vec{\gamma})=\left|\begin{array}{lll}\underline{a}_{11}+\gamma_{11} w_{11} & \underline{a}_{12}+\gamma_{12} w_{12} \\ \underline{a}_{12}+\gamma_{12} w_{12} & \underline{a}_{22}+\gamma_{22} w_{22}\end{array}\right|+\left|\begin{array}{lll}\underline{a}_{11}+\gamma_{11} w_{11} & \underline{a}_{13}+\gamma_{13} w_{13} \\ \underline{a}_{13}+\gamma_{13} w_{13} & \underline{a}_{33}+\gamma_{33} w_{33}\end{array}\right|$

$+\left|\begin{array}{ll}\underline{a}_{22}+\gamma_{22} w_{22} & \underline{a}_{23}+\gamma_{23} w_{23} \\ \underline{a}_{23}+\gamma_{23} w_{23} & \underline{a}_{33}+\gamma_{33} w_{33}\end{array}\right|$;

$a_{0}(\vec{\gamma})=\operatorname{det}(A(\vec{\gamma}))$.

3. Third case: if all elements of the matrix are independent and the eigenvalues are obtained from Eq. (3). For each $\gamma_{i j} \in\left[\begin{array}{ll}0 & 1\end{array}\right], w_{i j}=\bar{a}_{i j}-\underline{a}_{i j}, i, j=1,2,3$, we have the characteristic polynomial of degree 3 .

Note that if in all cases we have $\gamma_{i j}=0, i, j=1,2,3$ we have the deterministic case and for each $\gamma, \gamma_{i j} \in\left[\begin{array}{ll}0 & 1\end{array}\right], w_{i j}=\bar{a}_{i j}-\underline{a}_{i j}, i, j=1,2,3$, we need to get the sign of the roots for the characteristic polynomial of degree 3 to study the stability in (2). Here we want to choose $\gamma_{i j} \in\left[\begin{array}{ll}0 & 1\end{array}\right]$ so that there is an unique equilibrium [12]. 
Remark 1. For the square matrix of order 3, it is difficult to find, explicitly, the regions of the hypercube of dimension 9 that give a complete classification of the singularities of the characteristic matrix equation of system (2), even in the case of symmetry or the case of total dependence. Considering these, we will first describe the method called Cylindrical Algebraic Decomposition (CAD) used to find the lower and upper bound for real interval eigenvalue (see [1-3]).

To this end, we deal with a semi-algebraic set $S \subset \mathbb{R}^{n}$ which a finite union of sets defined by polynomial equations and inequalities with real coefficients. The CAD provides a partition of $S$ into semi-algebraic pieces which are homeomorphic to $] 01{ }^{i}$ for $i=1, \ldots, n$. Moreover, classification problems involving such a set $S$, reduces to the computing of a finite number of sample points in each connected component, and then facing a polynomial optimization question. The algorithm is implemented in RAGlib (Real Algebraic Geometry library) of the software Maple. For example, if $S=\left\{P_{1}=\ldots=P_{n}=0, \lambda_{1}>0, \ldots, \lambda_{m}>0\right\}$, the first step is a reduction to compute sample points in each component of $S$ defined with non-strict inequalities. There is a connected component $C_{e}$ of $S_{e}=\left\{P_{1}=\ldots=P_{n}=0, \lambda_{1} \geq e, \ldots, \lambda_{m} \geq 0,0<e<e_{0}\right\}$ and a suitable $e_{0}$ that can be found using notions of critical values and asymptotic critical values. The next step of the algorithm addresses an algebraic problem.

In the next example, we analyze a particular 3-dimensional interval differential system when all entries in the matrix are dependent and independent via CI. For the independent case, firstly we find conditions to get 3, 2 and 1 real eigenvalues and one real and a pair of complex as was described in the proof of the Theorem 1. Besides, in Proposition 1 and 2 we find the real interval eigenvalue by using techniques from real algebraic geometry. The same method cannot be used to find the complex interval eigenvalue, since the $\leq$ real ordering is not longer available. Finally, we compare the values obtained with the Deif's method [5] and Rohn's method [16].

Example 1. Consider the system of the differential equations $X^{\prime}(t)=A(\vec{\gamma}) X(t)$, where $A(\vec{\gamma})$ is an interval matrix written as

$$
A(\vec{\gamma})=\left(\begin{array}{ccc}
2 \gamma_{11} & 0 & -3+2 \gamma_{12} \\
-1+4 \gamma_{21} & -2+6 \gamma_{22} & -1+2 \gamma_{23} \\
-2+4 \gamma_{31} & 1 & 1+2 \gamma_{33}
\end{array}\right)
$$

Here to simplify the notation, $\gamma_{11}=\gamma_{1}, \gamma_{12}=0, \gamma_{13}=\gamma_{2}, \gamma_{21}=\gamma_{3}, \gamma_{22}=$ $\gamma_{4}, \gamma_{23}=\gamma_{5}, \gamma_{31}=\gamma_{6}, \gamma_{32}=0, \gamma_{33}=\gamma_{7}$ in such way $\vec{\gamma}=\left(\gamma_{1}, \gamma_{2}, \gamma_{3},=\right.$ $\left.\gamma_{4}, \gamma_{5}, \gamma_{6}, \gamma_{7}\right) . \operatorname{det}\left(A(\vec{\gamma})-\lambda I_{3}=0\right.$ implies that the characteristic polynomial

$$
P(\vec{\gamma})=a_{3}(\vec{\gamma}) \lambda^{3} \vec{\gamma}(\vec{\gamma})+a_{2}(\vec{\gamma}) \lambda^{2}(\vec{\gamma})+a_{1}(\vec{\gamma}) \lambda(\vec{\gamma})+a_{0}(\vec{\gamma})=0
$$

where $a_{3}(\vec{\gamma})=-1$;

$$
\begin{aligned}
& a_{2}(\vec{\gamma})=-1+2 \gamma_{1}+6 \gamma_{4}+2 \gamma_{7} ; \\
& a_{1}(\vec{\gamma})=7+2 \gamma_{1}-4 \gamma_{2}-6 \gamma_{4}-12 \gamma_{1} \gamma_{4}+2 \gamma_{5}-12 \gamma_{6}+8 \gamma_{2} \gamma_{6}+4 \gamma_{7}-4 \gamma_{1} \gamma_{7}-12 \gamma_{4} \gamma_{7} \\
& a_{0}(\vec{\gamma})=15-2 \gamma_{1}-10 \gamma_{2}-12 \gamma_{3}+8 \gamma_{2} \gamma_{3}-36 \gamma_{4}+12 \gamma_{1} \gamma_{4}+24 \gamma_{2} \gamma_{4}-4 \gamma_{1} \gamma_{5}-24 \gamma_{6}+ \\
& 16 \gamma_{2} \gamma_{6}+72 \gamma_{4} \gamma_{6}-48 \gamma_{2} \gamma_{4} \gamma_{6}-8 \gamma_{1} \gamma_{7}+24 \gamma_{1} \gamma_{4} \gamma_{7} .
\end{aligned}
$$


The solution of $(7), P(\vec{\gamma})=0$ for $\lambda$ subject to $\vec{\gamma}=\left(\gamma_{1}, \ldots, \gamma_{7}\right), 0 \leq \gamma_{i} \leq 1, i=1, \ldots, 7$ is obtained using the cube root formula

$$
\lambda(\vec{\gamma})=(P(\vec{\gamma}))^{\frac{1}{3}}, \vec{\gamma}=\left(\gamma_{1}, \ldots, \gamma_{7}\right)
$$

Thus, the real interval eigenvalue is

$$
[\lambda]=\left[\min _{0 \leq \gamma_{i} \leq 1}(P(\vec{\gamma}))^{\frac{1}{3}}, \min _{0 \leq \gamma_{i} \leq 1}(P(\vec{\gamma}))^{\frac{1}{3}}\right] .
$$

Firstly, consider in (7) that all interval entries in matrix are dependent, then $\gamma_{i}=$ $\gamma, \forall i=1, \ldots, 7$ and, we have the following equation for the eigenvalues:

$-\lambda^{3}(\gamma)+(1-10 \gamma) \lambda^{2}(\gamma)+\left(-20 \gamma^{2}-14 \gamma+7\right) \lambda(\gamma)+\left(-24 \gamma^{3}+120 \gamma^{2}-84 \gamma+15\right)=0$.

Thus, the eigenvalues are:

$$
\begin{aligned}
& \lambda_{1,2}(\gamma)=\left(\frac{1}{2} \pm \frac{\sqrt{3}}{2} i\right)\left\{\frac{2(\gamma-1) \sqrt{-128 \gamma^{4}-736 \gamma^{3}+2052 \gamma^{2}-1114 \gamma+69}}{\sqrt{27}}-\frac{224 \gamma^{3}-780 \gamma^{2}+726 \gamma-170}{27}\right\}^{1 / 3} \\
& +\left(\frac{\mp \sqrt{3}}{18} i-\frac{1}{18}\right) \sqrt[3]{\frac{\left(40 \gamma^{2}-62 \gamma+22\right)^{3}}{\frac{2(\gamma-1) \sqrt{-128 \gamma^{4}-736 \gamma^{3}+2052 \gamma^{2}-1114 \gamma+69}}{\sqrt{27}}-\frac{224 \gamma^{3}-780 \gamma^{2}+726 \gamma-170}{27}}-\frac{1-10 \gamma}{3}}
\end{aligned}
$$

and $\lambda_{3}(\gamma)$ is

$$
\begin{gathered}
\left\{\frac{2(\gamma-1) \sqrt{-128 \gamma^{4}-736 \gamma^{3}+2052 \gamma^{2}-1114 \gamma+69}}{\sqrt{27}}-\frac{224 \gamma^{3}-780 \gamma^{2}+726 \gamma-170}{27}\right\}^{1 / 3} \\
+\sqrt[3]{\frac{40 \gamma^{2}-62 \gamma+22}{\frac{2(\gamma-1) \sqrt{-128 \gamma^{4}-736 \gamma^{3}+2052 \gamma^{2}-1114 \gamma+69}}{\sqrt{27}}-\frac{224 \gamma^{3}-780 \gamma^{2}+726 \gamma-170}{27}}}
\end{gathered}
$$

For $\gamma=0, \lambda_{3}(0)=3, \lambda_{1,2}(0)=-2 \pm i ; \gamma=1, \lambda_{1,2,3}=3, \gamma=.07098016326$ $\left.152795, \lambda_{3} .07098016326152795\right)\left(=2.89667, \lambda_{1,2}=-1.59343 \pm 0.886988 i ; \gamma=\right.$ $.6768941902714837, \lambda_{1,2}(.07098016326152795)=2.53294 \pm 1.28951 i, \lambda_{3}=0.703058$, and so on.

Analysing the graph of

$$
\left\{\frac{2(\gamma-1) \sqrt{-128 \gamma^{4}-736 \gamma^{3}+2052 \gamma^{2}-1114 \gamma+69}}{\sqrt{27}}-\frac{224 \gamma^{3}-780 \gamma^{2}+726 \gamma-170}{27}\right\}^{1 / 3},
$$

we can conclude that for $\gamma$ between 0.29 and 0.39 we have three real eigenvalues, for $\gamma=1$ there is a unique triple real root and in other cases we have one real and two complex eigenvalues.

Secondly, consider $0 \leq \gamma_{i} \leq 1, i=1, \ldots, 7$ are independent, then can be proved that for the real case, the $\mathrm{min} / \max$ of the eigenvalues are obtained at a corner point on the boundary of the space of the parameters in a hypercube of the 7-dimension. Note that to obtain conditions for the complex eigenvalues is not easy, because the complex set is not an ordered set and we have an optimization problem to find the conditions for the parameters $\gamma_{i}, i=1, \ldots, 7$. For all $\gamma_{i} \in \mathbb{R}^{7}$, the $3 \times 3$ matrix $A(\vec{\gamma})$ has at least one real eigenvalue, therefore for all $\vec{\gamma} \in \mathbb{R}^{7}$, one can define $\lambda_{\min }(A(\vec{\gamma}))$ 
(resp. $\lambda_{\max }(A(\vec{\gamma}))$ as the minimal (resp. maximal) real eigenvalue of $A(\vec{\gamma})=$ $A\left(\gamma_{1}, \ldots, \gamma_{7}\right)$. Let us now consider the compact set $\mathcal{B}_{7}=[0,1]^{7} \subset \mathbb{R}^{7}$.

We denote by $P=P(\lambda, \vec{\gamma})$ the characteristic polynomial of $A(\vec{\gamma})$. Then by considering the discriminant $(5)$, where $q(\vec{\gamma})=a_{0}(\vec{\gamma})+\frac{a_{2}(\vec{\gamma}) a_{1}(\vec{\gamma})}{3}+\frac{2}{27} a_{2}^{3}(\vec{\gamma})$ and $p(\vec{\gamma})=-\frac{1}{3} a_{1}(\vec{\gamma})-\frac{1}{3} a_{2}^{2}(\vec{\gamma})$ we have the following analysis.

1. There are three different real eigenvalues for $\triangle=[-162.963,0]$. The left side is defined by parameters $\gamma_{4}=\gamma_{5}=\gamma_{7}=1, \gamma_{1}=\gamma_{2}=\gamma_{3}=\gamma_{6}=0$ that define the characteristic equation $-\lambda^{3}+7 \lambda^{2}-5 \lambda-21=-(\lambda-3)(\lambda-5.31)(\lambda-1.32)=0$ and the right side by $\gamma_{1}=0.64, \gamma_{2}=0.38, \gamma_{3}=0, \gamma_{4}=.25, \gamma_{5}=.62 ; \gamma_{6}=0.64, \gamma_{7}=0.33$ such that $-\lambda^{3}+2.44 \lambda^{2}-1.6992 \lambda-0.2432=-(\lambda-.198725)(\lambda-0.941681)(\lambda-$ $1.29959)=0$. We have that the equilibrium point in this case are saddle and repulsor, respectively.

2. There is one real and a complex conjugate for $\triangle=\left[\begin{array}{ll}0 & 452\end{array}\right]$ The left side is defined by parameters $\gamma_{4}=0.75, \gamma_{5}=0.12, \gamma_{7}=.52, \gamma_{1}=0.89, \gamma_{2}=0.92, \gamma_{3}=0, \gamma_{6}=.18$ that define the characteristic equation $-\lambda^{3}+6.32 \lambda^{2}-12.4564 \lambda+7.8788=-(\lambda-$ $3.18777)(\lambda-1.56612+0.137312 i)(\lambda-1.56612-0.137312 i)=0$ and the right side by $\gamma_{1}=\gamma_{2}=\gamma_{3}=\gamma_{7}=0, \gamma_{4}=\gamma_{5} \gamma_{6}=1$, such that $-\lambda^{3}+5 \lambda^{2}-9 \lambda+27=$ $-(\lambda-0.321648+2.46858 i)(\lambda-0.321648-2.46858 i)(\lambda-4.3567)=0$. Note that the equilibrium point in both cases are repulsing.

3. If $\triangle(\vec{\gamma})=0$ and $p(\vec{\gamma})<0$, then (7) has 3 real roots, where two them are equal. Then,

$$
\begin{aligned}
& \triangle(\vec{\gamma})=\left(15-2 \gamma_{1}-10 \gamma_{2}-12 \gamma_{3}+8 \gamma_{2} \gamma_{3}-36 \gamma_{4}+12 \gamma_{1} \gamma_{4}+24 \gamma_{2} \gamma_{4}-4 \gamma_{1} \gamma_{5}-24 \gamma_{6}\right. \\
& \quad+16 \gamma_{2} \gamma_{6}+72 \gamma_{4} \gamma_{6}-48 \gamma_{2} \gamma_{4} \gamma_{6}-8 \gamma_{1} \gamma_{7}+24 \gamma_{1} \gamma_{4} \gamma_{7}+\frac{1}{3}\left(7+2 \gamma_{1}-4 \gamma_{2}-6 \gamma_{4}\right. \\
& \left.\quad-12 \gamma_{1} \gamma_{4}+2 \gamma_{5}-12 \gamma_{6}+8 \gamma_{2} \gamma_{6}+4 \gamma_{7}-4 \gamma_{1} \gamma_{7}-12 \gamma_{4} \gamma_{7}\right)\left(-1+2 \gamma_{1}+6 \gamma_{4}+2 \gamma_{7}\right) \\
& \left.\quad+\quad \frac{2}{27}\left(-1+2 \gamma_{1}+6 \gamma_{4}+2 \gamma_{7}\right)^{3}\right)^{2}+4\left(-\frac{1}{3}\left(7+2 \gamma_{1}-4 \gamma_{2}-6 \gamma_{4}-12 \gamma_{1} \gamma_{4}+2 \gamma_{5}\right.\right. \\
& \left.\left.-12 \gamma_{6}+8 \gamma_{2} \gamma_{6}+4 \gamma_{7}-4 \gamma_{1} \gamma_{7}-12 \gamma_{4} \gamma_{7}+\frac{1}{3}\left(-1+2 \gamma_{1}+6 \gamma_{4}+2 \gamma_{7}\right)^{2}\right)\right)^{3}
\end{aligned}
$$

and $p(\vec{\gamma})<0$. (7) has two equal roots if, and only if, (7) and its first derivative have the same roots. Then we have the condition $\gamma_{i}=0, i=1,2,7$. Moreover, if $\gamma_{i}=0, i=2,5$, we have the condition $\gamma_{6}<\frac{11}{16}$. Then for example $\gamma_{6}=\frac{1}{2}$, then $\gamma_{3}=\frac{43}{16}$ or $\frac{1}{6}$, with characteristic equation given by $-\frac{5}{27}+\lambda-\lambda^{2}-\lambda^{3}=$ $-\left(\lambda-\frac{1}{3}\right)^{2}\left(\lambda+\frac{5}{3}\right)=0$ and $1+\lambda-\lambda^{2}-\lambda^{3}=-(\lambda+1)^{2}(\lambda-1)=0$, respectively. If $p(\vec{\gamma})=q(\vec{\gamma})=0$, that is, $a_{0}(\vec{\gamma})=\frac{25}{27} a_{2}^{3}(\vec{\gamma})$, (7) has one triple real root. The expression is

$$
\begin{aligned}
& \gamma_{2}=\left(-215+102 \gamma_{1}-150 \gamma_{1}^{2}+100 \gamma_{1}^{3}+162 \gamma_{3}+711 \gamma_{4}-1062 \gamma_{1} \gamma_{4}+900 \gamma_{1}^{2} \gamma_{4}\right. \\
& -1350 \gamma_{4}^{2}+2700 \gamma_{1} \gamma_{4}^{2}+2700 \gamma_{4}^{3}+54 \gamma_{1} \gamma_{5}+324 \gamma_{6}-972 \gamma_{4} \gamma_{6}+75 \gamma_{7}-192 \gamma_{1} \gamma_{7} \\
& +300 \gamma_{1}^{2} \gamma_{7}-900 \gamma_{4} \gamma_{7}+1476 \gamma_{1} \gamma_{4} \gamma_{7}+2700 \gamma_{4}^{2} \gamma_{7}-150 \gamma_{7}^{2}+300 \gamma_{1} \gamma_{7}^{2}+900 \gamma_{4} \gamma_{7}^{2} \\
& \left.+100 \gamma_{7}^{3}\right) / 27\left(-5+4 \gamma_{3}+12 \gamma_{4}+8 \gamma_{6}-24 \gamma_{4} \gamma_{6}\right)
\end{aligned}
$$


where $0 \leq \gamma_{i} \leq 1, i=1, \ldots, 7$. For example, if $\gamma_{i}=1, \forall i=1, \ldots, 7, \lambda=3$ is the unique eigenvalue.

Equation (5) is equivalent to $4 q^{3}(\vec{\gamma})+27 p^{2}(\vec{\gamma})$ and to $\frac{q^{2}}{4}(\vec{\gamma})+\frac{p^{3}(\vec{\gamma})}{27}$, but to find the roots of (7) we need to use the last one [4], so that

$$
\left[\frac{1}{4}\left(\frac{2}{27} a_{2}(\vec{\gamma})^{2}+\frac{a_{2}(\vec{\gamma}) a_{1}(\vec{\gamma})}{3}+a_{0}(\vec{\gamma})\right)^{2}+\frac{1}{27}\left(\frac{-a_{2}(\vec{\gamma})^{2}}{3}-a_{1}\right)^{3}\right]
$$

Our first goal is to estimate the extreme values of the multiple roots in $\mathcal{B}_{7}$. We begin by dealing with the multiple roots of the derivative $\frac{\partial P}{\partial \lambda}$ of the characteristic polynomial. We observe that the parameter $\gamma_{3}$ is not present in this derivative. Let us denote $\vec{\gamma}=\left(\gamma_{1}, \gamma_{2}, \gamma_{4}, \gamma_{5}, \gamma_{6}, \gamma_{7}\right)$. Let

$$
P_{1}(\vec{\gamma})=\frac{\partial P}{\partial \lambda}(\lambda, \vec{\gamma})=3 \lambda^{2}+2 a_{2}(\vec{\gamma}) \lambda+a_{1}(\vec{\gamma})
$$

In the Eq. (11,) let us now consider the compact set $\vec{\gamma} \in \mathcal{B}_{6}=[0,1]^{6} \subset \mathbb{R}^{6}$. Then, $P(\lambda, \vec{\gamma})$ has a double root in an interior point of $\mathcal{B}_{6}$ if for

$\lambda=\frac{-a_{2}(\vec{\gamma}) \pm \sqrt{a_{2}^{2}(\vec{\gamma})-3 a_{1}(\vec{\gamma})}}{3}$, we have $a_{2}^{2}(\vec{\gamma})-3 a_{1}(\vec{\gamma})=0$ and in this case $\lambda=\frac{-a_{2}(\vec{\gamma})}{3}$.

The real eigenvalues can be shown to be in $[-3.395 .69]$ in accordance to the Propositions 1 and 2, which follow.

Third, Deif [5] considers the interval matrix $\left(\begin{array}{ccc}{\left[\begin{array}{ll}0 & 2\end{array}\right]} & {\left[\begin{array}{ll}0 & 0\end{array}\right]} & {\left[\begin{array}{cc}-3 & -1\end{array}\right]} \\ {\left[\begin{array}{ll}-1 & 3\end{array}\right]\left[\begin{array}{ll}-2 & 4\end{array}\right]} & {\left[\begin{array}{ll}-1 & 1\end{array}\right]} \\ {\left[\begin{array}{ll}-2 & 2\end{array}\right]} & {\left[\begin{array}{ll}1 & 1\end{array}\right]} & {\left[\begin{array}{ll}1 & 3\end{array}\right]}\end{array}\right)$ and found that $\operatorname{Re}(\lambda) \in\left[\begin{array}{ll}0.2873 & 4.7346\end{array}\right]$ and $\operatorname{Im}(\lambda) \in\left[\begin{array}{ll}0 & 2.1754\end{array}\right]$.

Fourth, by using the Rohn's Method outlined in [12], we found $\operatorname{Re}(\lambda) \in$ $\left[\begin{array}{ll}-2.70 & 5.27\end{array}\right]$ and $\operatorname{Im}(\lambda) \in\left[\begin{array}{ll}-5.34544 & 5.34544\end{array}\right]$.

However, in the method using CI, the matrix $\left(\begin{array}{ccc}0 & 0 & 0 \\ 3 & -2 & 1 \\ -2 & 1 & 1\end{array}\right)$ and $\left(\begin{array}{ccc}2 & 0 & -3 \\ 3 & -2 & 1 \\ -2 & 1 & 1\end{array}\right)$ give us real eigenvalues in the interval [ -3.39 5.69].

In the complex case, we find numerically, $\operatorname{Re}(\lambda) \in[-2.113 .36429]$ for $\gamma_{i}=0, \forall i \neq 5$ and $\gamma_{1}=0.94, \gamma_{i}=1, i \neq 1,5, \gamma_{5}=0$, respectively (Fig. 2).

The Propositions 1 and 2 proof that the real interval eigenvalue is [-3.39 5.69]. Firstly, it is necessary to find a value $\Lambda$ such that for all $\lambda>\Lambda, F_{\lambda}=$ $\left\{\gamma \in \operatorname{int}\left(B_{7}\right) \mid \lambda_{M}(\gamma)=\lambda\right\}$ does not intersects the discriminant of $P(\lambda, \vec{\gamma})$. 


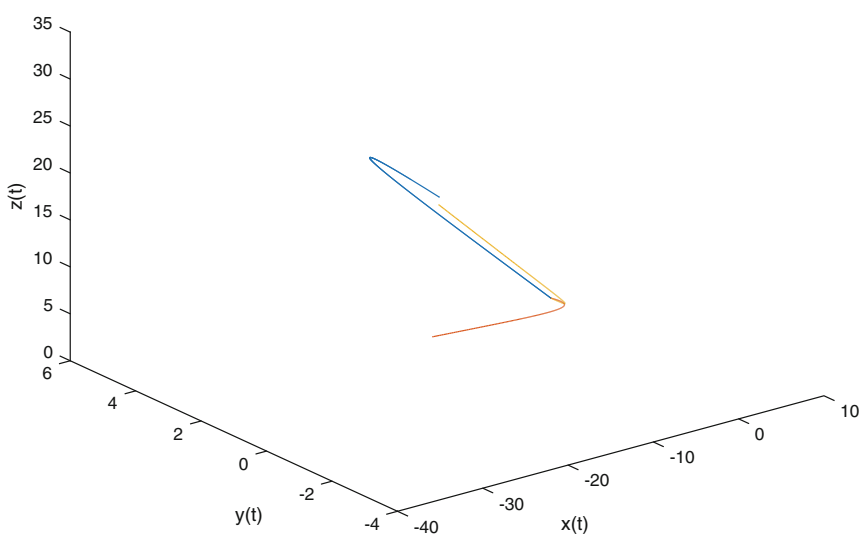

Fig. 2. Trajectories solutions for $\gamma_{i}=3 / 5,1 / 5, i=1, \ldots, 7$ and $\gamma_{i}=1 / 5, i \neq 6, \gamma_{6}=$ $3 / 5$, respectively.

Proposition 1. $\lambda_{\max }$ is the upper bound for the real interval eigenvalue in (7) and is the greatest root of $\lambda^{3}-9 \lambda^{2}+19 \lambda-1$, obtained from the Eq. (7) for $\vec{\gamma}=\left(\begin{array}{lllllll}1 & 0 & 0 & 1 & 1 & 0 & 1\end{array}\right)$.

Proof. Let $V_{5}^{+}=\left\{\vec{\gamma} \in \mathcal{B}_{7} \mid \lambda(\vec{\gamma})>5\right\}$. First, we show that $V_{5}^{+}$is non-empty. Note that for $\vec{\gamma}^{0}=(1,1,0,1,1,0,1)$ we have $P\left(\lambda, \vec{\gamma}^{0}\right)=(\lambda-5)(\lambda-1)(\lambda-3)$ such that $\lambda(\vec{\gamma})=5$. By taking $\vec{\gamma}^{\varepsilon}=(1-\varepsilon, 1-8 \varepsilon, \varepsilon, 1-\varepsilon, 1-\varepsilon, 1-\varepsilon), \varepsilon>0$ sufficiently small, one gets $\lambda\left(\vec{\gamma}^{\varepsilon}\right)=5+\frac{\varepsilon}{4}+o(\varepsilon)>5$ with $\vec{\gamma}^{\varepsilon} \in \operatorname{int}\left(\mathcal{B}_{7}\right)$. Then, the maximum is not attained at an interior set.

We consider on $V_{5}^{+}$a function $\lambda: \vec{\gamma} \mapsto \lambda(\vec{\gamma})$. Since $\lambda(\vec{\gamma})$ is smooth on $V_{5}^{+}$, $\frac{\partial P}{\partial \lambda(\vec{\gamma})}(\lambda(\vec{\gamma}), \vec{\gamma}) \neq 0$

The gradient $\vec{\nabla} \vec{\gamma}(P)$ of $P$ with respect to $\vec{\gamma}$ is such that:

$$
\vec{\nabla}_{\vec{\gamma}}(P(\lambda(\vec{\gamma}), \vec{\gamma}))=-\frac{\partial P}{\partial \lambda(\vec{\gamma})}(\lambda(\vec{\gamma}), \vec{\gamma}) \cdot \vec{\nabla}_{\vec{\gamma}} \lambda(\vec{\gamma})
$$

Moreover, $\lambda=\lambda(\vec{\gamma})$ is the greatest root of $P(\lambda, \vec{\gamma})$, which is a degree 3 polynomial with positive leading coefficient. Hence $\frac{\partial P}{\partial \lambda}(\lambda(\vec{\gamma}), \vec{\gamma})>0$. It follows that the respective coordinates of $\vec{\nabla} \vec{\gamma} \lambda(\vec{\gamma})$ and $\vec{\nabla} \underset{\gamma}{ } P(\lambda(\vec{\gamma}), \vec{\gamma})$ have opposite signs. 
The transposed gradient ${ }^{t} \vec{\nabla} \underset{\vec{\gamma}}{ } P(x, \vec{\gamma})$ is

$$
\left(\begin{array}{c}
\frac{\partial P}{\partial \gamma_{1}}(\lambda(\vec{\gamma}), \vec{\gamma}) \\
\frac{\partial P}{\partial \gamma_{2}}(\lambda(\vec{\gamma}), \vec{\gamma}) \\
4\left(3-2 \gamma_{2}\right) \\
\frac{\partial P}{\partial \gamma_{4}}(\lambda(\vec{\gamma}), \vec{\gamma}) \\
2\left(2 \gamma_{1}-\lambda\right) \\
4\left(3-2 \gamma_{2}\right)\left(\lambda-6 \gamma_{4}+2\right) \\
2\left(\lambda-6 \gamma_{4}+2\right)\left(2 \gamma_{1}-\lambda\right)
\end{array}\right)
$$

Let us consider a point $\vec{\gamma} \in V_{5}^{+}, \lambda(\vec{\gamma})>5$ and $0 \leq \gamma_{i} \leq 1, i=1, \ldots, 7$, then the signs of the coordinates for $\vec{\nabla} \vec{\gamma}(P(\lambda(\vec{\gamma}), \vec{\gamma}))$ are $[-,-,+,-,-,+,-]$. The strategy to find the maximum eigenvalue is to choose one direction for the gradient, considering one constant, to find the directions where it is increasing, because we need to build the trajectory as a piecewise function in hypercube $\mathcal{B}_{7}$. Then we consider the smooth vector field $\chi$ defined on $V_{5}^{+}$,

$$
\chi(\vec{\gamma})=\left(\begin{array}{c}
0 \\
0 \\
4\left(3-2 \gamma_{2}\right) \\
0 \\
2\left(2 \gamma_{1}-\lambda(\vec{\gamma})\right. \\
4\left(3-2 \gamma_{2}\right)\left(\xi(\Gamma)-6 \gamma_{4}+2\right) \\
2\left(\xi(\Gamma)-6 \gamma_{4}+2\right)\left(2 \gamma_{1}-\lambda(\vec{\gamma})\right) .
\end{array}\right)
$$

Let $\lambda(\vec{\gamma})^{0} \in V_{5}^{+} \cap \operatorname{int}\left(\mathcal{B}_{7}\right)$ and $\varphi: t \mapsto \varphi(t)$ be the trajectory of $\chi$ such that $\varphi(0)=\lambda(\vec{\gamma})^{0}$. Along this trajectory $\xi(\varphi(t))$ strictly increases, hence $\varphi(t)$ remains in $V_{5}^{+}$. Let $\psi(\lambda(\vec{\gamma}))=\gamma_{3}, \gamma_{6}\left(1-\gamma_{5}\right)\left(1-\gamma_{7}\right)$. For all $\lambda\left(\vec{\gamma} \in \operatorname{int}\left(\mathcal{B}_{7}\right)\right.$, we have $\operatorname{sign}\left(\frac{\partial \psi}{\partial \gamma_{3}}\right)=\operatorname{sign}\left(\frac{\partial \psi}{\partial \gamma_{6}}\right)>0$ and $\operatorname{sign}\left(\frac{\partial \psi}{\partial \gamma_{5}}\right)=\operatorname{sign}\left(\frac{\partial \psi}{\partial \gamma_{7}}\right)<0$. In addition $V_{5}^{+}$, $\vec{\nabla}_{\lambda(\vec{\gamma})}(P(\lambda(\vec{\gamma}), \lambda(\vec{\gamma})))$ and $\vec{\nabla}_{\Gamma} \psi(\Gamma)$ have exactly the same signs, excepting where the sign does not change, as well as $\lambda(\lambda(\vec{\gamma}))$ and $\vec{\nabla}_{\lambda(\vec{\gamma})} \psi(\lambda(\vec{\gamma}))$.

It follows that $t \mapsto \psi(\varphi(t))$ decreases, so that it cannot be the minimum on $\operatorname{int}\left(\mathcal{B}_{7}\right)$ and there exists a minimal $t^{1}>0$ such that $\psi\left(\varphi\left(t^{1}\right)\right)=0$. Furthermore, if one puts $Z(\psi)=\left\{\lambda(\vec{\gamma}) \in \mathcal{B}_{7} \mid \psi(\lambda(\vec{\gamma}))=0\right\}$, this proves that $\lambda_{\max }=$ $\max _{\lambda(\vec{\gamma}) \in\left(Z(\psi) \cap \mathcal{B}_{7}\right)}\{\lambda(\vec{\gamma})\}$.

We decrease/increase iteratively each coordinate $\left\{\gamma_{3}, \gamma_{6}, \gamma_{5}, \gamma_{7}\right\}$ to 0 or 1 accordingly to the (constant, non-zero) corresponding gradient coordinate sign. We finally get a point $\gamma^{k} \in \partial \mathcal{B}_{7}(k \geq 1)$ such that $\left\{\gamma_{5}^{k}=\gamma_{7}^{k}=1, \gamma_{3}^{k}=\gamma_{6}^{k}=0\right\}$ and $\lambda\left(\vec{\gamma}^{k}\right)>\lambda\left(\vec{\gamma}^{0}\right)$. Observe that $\gamma_{1}^{k}=\gamma_{1}^{0}, \gamma_{2}^{k}=\gamma_{2}^{0}, \gamma_{4}^{k}=\gamma_{4}^{0}$.

We now have to deal with the remaining free coordinates $\left(\gamma_{1}, \gamma_{2}, \gamma_{4}\right)$. So let us consider the polynomial

$$
\tilde{P}\left(\lambda,\left(\gamma_{1}, \gamma_{2}, \gamma_{4}\right)\right)=P\left(\lambda,\left(\gamma_{1}, \gamma_{2}, 0, \gamma_{4}, 1,0,1\right)\right)
$$

with $\left(\gamma_{1}, \gamma_{2}, \gamma_{4}\right) \in \mathcal{B}_{3}=[0,1]^{3}$. The problem we are facing is exactly the same as the original one for $P$. For all $h=\left(\gamma_{1}, \gamma_{2}, \gamma_{4}\right)$, denote by $\tilde{\lambda}(h)=$ 
$\left.\lambda\left(\gamma_{1}, \gamma_{2}, 0, \gamma_{4}, 1,0,1\right)\right)$ the greatest root of $\tilde{P}(\lambda, h)$. We introduce the non-empty semi-algebraic subset:

$$
\tilde{V}_{5}^{+}=\left\{\left(\gamma_{1}, \gamma_{2}, \gamma_{4}\right) \in \mathcal{B}_{3} \mid\left(\gamma_{1}, \gamma_{2}, 0, \gamma_{4}, 1,0,1\right) \in V_{5}^{+}\right\}
$$

For all $h=\left(\gamma_{1}, \gamma_{2}, \gamma_{4}\right) \in \mathcal{B}_{3}$, and all $\lambda_{1} \in \mathbb{R}$, the gradient $\vec{\nabla}{ }_{h} \tilde{P}\left(\lambda_{1}, h\right)$ equals $\left(\frac{\partial P}{\partial \gamma_{1}}\left(\lambda_{1}, \lambda(\vec{\gamma})\right), \frac{\partial P}{\partial \gamma_{2}}\left(\lambda_{1}, \Gamma\right), \frac{\partial P}{\partial \gamma_{4}}\left(\lambda_{1}, \lambda(\vec{\gamma})\right)\right)$ where $\lambda(\vec{\gamma})=\left(\gamma_{1}, \gamma_{2}, 0, \gamma_{4}, 1,0,1\right)$ $\in V_{5}^{+}$, then $\tilde{\lambda}((\vec{\gamma})>5$, such that

$-\frac{\partial \tilde{P}}{\partial \gamma_{2}}(\tilde{\xi}(h), h)>0$.

$-\frac{\partial \tilde{P}}{\partial \gamma_{1}}(\tilde{\xi}(h), h)<0$.

$-\frac{\partial \tilde{P}}{\partial \gamma_{4}}(\tilde{\xi}(h), h)<0$.

We integrate $\vec{\nabla}_{h} \tilde{P}$ from a point $h^{0} \in \mathcal{B}_{3}$ and get after at most two other iterations, a point $h^{*}$ such that $\left\{\gamma_{1}=\gamma_{4}=1, \gamma_{2}=0\right\}$, with $\tilde{\lambda}\left(h^{*}\right)>\tilde{\lambda}\left(h^{0}\right)$. To $h^{*} \in \mathcal{B}_{3}$ corresponds the unique point $\vec{\gamma}^{*} \in \mathcal{B}_{7}$ such that $\left\{\gamma_{2}=\gamma_{3}=\gamma_{6}=\right.$ $\left.0, \gamma_{1}=\gamma_{4}=\gamma_{5}=\gamma_{7}=1\right\}$. Moreover $\lambda\left(\vec{\gamma}^{*}\right)>\lambda\left(\left(\vec{\gamma}^{k}\right)>\lambda\left(\vec{\gamma}^{0}\right)\right.$.

This results in $P\left(\lambda, \vec{\gamma}^{*}\right)=\lambda^{3}-9 \lambda^{2}+19 \lambda-1$ and one can compute explicitly the value $\lambda\left(\vec{\gamma}^{*}\right)$ which is realized at $\lambda_{\max }=5.69$.

Proposition 2. $\lambda_{\min }=-3.39$ is the lower bound for the real interval eigenvalue in (7) and is the smallest root of $X^{3}+X^{2}-9 X-3$, obtained from the Eq. (7) for $\vec{\gamma}=\left(\begin{array}{lllllll}0 & 0 & 1 & 0 & 1 & 0 & 0\end{array}\right)$.

Proof. The proof is similar to the proof of Proposition 1.

Remark 2. Therefore, in this example we showed:

1. Given an equation

$$
P_{n}(x)=x^{x}+a_{n-1} x^{n-1}+\ldots+a_{1} x+a_{0}=0,
$$

The discriminant $\Delta\left(P_{n}\right)$ of $P_{n}$ is a polynomial in the indeterminates $\left(a_{n-1}, \ldots, a_{1}, a_{0}\right)$ with integer coefficients (explicitly computed as the determinant of a Sylvester matrix, see [13]). The set $\left\{\left(a_{n-1}, \ldots, a_{1}, a_{0}\right) \mid P_{n}(x)=\right.$ 0 has root with multiplicity $\}$ is exactly the set $\left\{\left(a_{n-1}, \ldots, a_{1}, a_{0}\right) \Delta\left(P_{n}\right)=\right.$ $0\}$. Then, varying the coefficients in (15) we can get what type of roots it has. In particular the condition on the discriminant for $n=3$ defines the type of roots for the polynomial equation when $0 \leq \gamma_{i} \leq 1, i=1, \ldots, 7$ in (7) are varying. In the real case, we have methods to find them, but in the complex case it is not easy to characterize them completely;

2. There are other methods to find the bounds for interval eigenvalue for (7), but it is not easy(in general not possible) to define the matrix by choosing the entries in interval matrix to get the corresponding eigenvalues. That is, once has the $\max / \min$ eigenvalues the matrix that generated these eigenvalues is impossible to find. This is not true with the CI approach. We always know the matrix that generated the eigenvalues; 
3. CIA gives us the option to choose the parameters for each eigenvalue in the way we can find the matrix explicitly, but may be a NP-hard procedure;

4. Many authors consider the interval matrix $[A]=\left[A_{c}-\triangle A A_{c}+\triangle A\right]=$ $[\underline{A} \bar{A}]$. By CIA, we get $A_{c}-\triangle A$ for $\gamma_{i}=0$ and $A_{c}+\triangle A$ for $\gamma_{i}=1$.

5. $A_{c}$ is obtained from CIA taking $\gamma_{i}=\frac{1}{2}$, but for the element $[-3-1], \triangle a_{13}=$ $\frac{-1-(-3)}{2}=1$ and by CIA $a_{13}\left(\gamma_{2}\right)=-3+2 \gamma_{2}$, then $-3+2 \gamma_{2}=1$ if $\gamma_{2}=2$. This means that methods used by Deif [5], Rohn [16] and [9] are not equivalent. Note the elements $\triangle a_{13}=1 \notin[-3-1]$ neither $\triangle a_{32}=0 \notin\left[\begin{array}{ll}1 & 1\end{array}\right]$.

6. Mathematica and Maple were used as tools to analyze and get some results.

\section{Conclusion}

This research outlined a method, involving semi algebraic sets theory, for which the stability of interval linear differential equations can be analyzed via constraint intervals. As a by product, a method for obtaining conditions about parameters to get real or complex eigenvalues of interval matrices of order $3 \times 3$ were developed.

\section{References}

1. Benedetti, R., Risler, J.J.: Real Algebraic and Semi-algebraic sets, Actualiteés mathematique. Herman, Berlin (1990)

2. Bonnard, B., Cotes, O., Faugère, J.C., Jacquemard, A., Rouot, M.S., Verron, T.: Algebraic geometric classification of the singular flow in the contrast imaging problem in nuclear magnetic resonance. https://hal.inria.fr/hal-01556806. Accessed 4 Feb 2020

3. Bonnard, B., Faugère, J.C., Jacquemard A., Safey El Din, M., Verron, T.: Determinantal sets, singularities and application to optimal control in medical imagery. In: Proceedings of the ISSAC, pp. 103-110 (2016)

4. Burnside, W.S., Panton, W.A.: The Theory of Equations: With an Introduction to the Theory of Binary Algebraic Forms. Dublin University Press Series. Longmans Green, London (1802)

5. Deif, A.S.: The interval eigenvalue problem. Z. Angew. Math. Mech. 71, 61-64 (1991)

6. Hladík, M., Daney, D., Tsigaridas, E.: An algorithm for addressing the real interval eigenvalue problem. J. Comput. Appl. Math. 235, 2715-2730 (2010)

7. Hladík, M., Daney, D., Tsigaridas, E.: A filtering method for the interval eigenvalue problem. Appl. Math. Comput. 217, 5236-5242 (2011)

8. Hladík, M., Daney, D., Tsigaridas, E.: Bounds on real eigenvalues and singular values of interval matrices. SIAM J. Matrix Anal. Appl. 31(4), 2116-2129 (2010)

9. Hladík, M.: Bounds on eigenvalues of real and complex interval matrices. Appl. Math. Comput. 219(10), 5584-5591 (2013)

10. Leng, H., He, Z., Yuan, Q.: Computing bounds to real eigenvalues of real-interval matrices. Int. J. Numer. Methods Eng. 74, 523-530 (2008)

11. Lodwick, W.A.: Constrained interval arithmetic. CCM Report 138, University of Colorado (USA), February 1999 
12. Mizukoshi, M.T., Lodwick, W.A.: The interval eigenvalue problem using constraint interval analysis with an application to linear differential equations: a first step toward the fuzzy eigenvalue problem. Fuzzy Sets and Systems (2019, submitted)

13. Mignotte, M.: Mathematics for Computer Algebra. Springer, New York (1992). https://doi.org/10.1007/978-1-4613-9171-5

14. Qiu, Z., Müller, P.C., Frommer, A.: An approximate method for the standard interval eigenvalue problem of real non-symmetric interval matrices. Commun. Numer. Methods Eng. 17, 239-251 (2001)

15. Rohn, J., Deif, A.: On the range of an interval matrix. Computing 47, 373-377 (1992)

16. Rohn, J.: Interval matrices: singularity and real eigenvalues. SIAM J. Matrix Anal. Appl. 14, 82-91 (1993) 